\title{
Effects of psychological eating behaviour domains on the association between socio-economic status and BMI
}

Antje Löffler ${ }^{1,2,3, *}$, Tobias Luck ${ }^{1,2}$, Francisca S Then ${ }^{1,2}$, Claudia Luck-Sikorski ${ }^{1,4,5}$ Alexander Pabst ${ }^{1}$, Peter Kovacs ${ }^{4}$, Yvonne Böttcher ${ }^{4,6}$, Jana Breitfeld ${ }^{4}$, Anke Tönjes ${ }^{7}$, Annette Horstmann ${ }^{4,8}$, Markus Löffler ${ }^{9}$, Christoph Engel ${ }^{9}$, Joachim Thiery ${ }^{10}$, Arno Villringer ${ }^{8,11}$, Michael Stumvoll ${ }^{4}$ and Steffi G Riedel-Heller ${ }^{1}$

${ }^{1}$ Institute of Social Medicine, Occupational Health and Public Health (ISAP), University of Leipzig, Leipzig, Germany: ${ }^{2}$ LIFE-Leipzig Research Center for Civilization Diseases, University of Leipzig, Leipzig, Germany: ${ }^{3}$ Brandenburg University of Technology, Department of Public Health, Universitätsplatz 1, 01968 Senftenberg, Germany: ${ }^{4}$ IFB Adiposity Diseases, Leipzig University Medical Center, Leipzig, Germany: ${ }^{5}$ University of Applied Health Sciences, Gera, Germany: 'Institute of Clinical Medicine, Section for Clinical Molecular Biology, University of Oslo, Oslo, Norway: ${ }^{7}$ Division of Endocrinology and Nephrology, Medical Department, University of Leipzig, Leipzig, Germany:

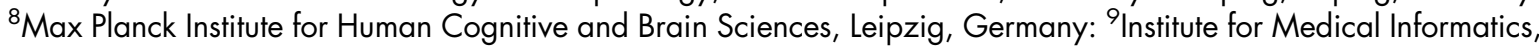
Statistics and Epidemiology (IMISE), University of Leipzig, Leipzig, Germany: ${ }^{10}$ Institute of Laboratory Medicine, Clinical Chemistry and Molecular Diagnostics, University of Leipzig, Leipzig, Germany: ${ }^{1}$ Day Clinic of Cognitive Neurology, University of Leipzig, Leipzig, Germany

Submitted 16 December 2016: Final revision received 5 June 2017: Accepted 12 June 2017: First published online 24 July 2017

\begin{abstract}
Objective: The current study investigates potential pathways from socio-economic status (SES) to BMI in the adult population, considering psychological domains of eating behaviour (restrained eating, uncontrolled eating, emotional eating) as potential mediators stratified for sex.

Design: Data were derived from the population-based cross-sectional LIFE-AdultStudy. Parallel-mediation models were conducted to obtain the total, direct and indirect effects of psychological eating behaviour domains on the association between SES and BMI for men and for women.

Setting: Leipzig, Germany.

Subjects: We studied 5935 participants aged 18 to 79 years.

Results: Uncontrolled eating mediated the association between SES and BMI in men only and restrained eating in both men and women. Emotional eating did not act as mediator in this relationship. The total effect of eating behaviour domains on the association between SES and BMI was estimated as $\beta=-0.03$ (se $0.02 ; 95 \% \mathrm{CI}-0.062,-0.003)$ in men and $\beta=-0.18$ (se $0.02 ; 95 \% \mathrm{CI}-0.217$, $-0 \cdot 138)$ in women.

Conclusions: Our findings do not indicate a strong overall mediation effect of the eating behaviour domains restrained eating, uncontrolled eating and emotional eating on the association between SES and BMI. Further research on other pathways of this association is strongly recommended. Importantly, our findings indicate that, independent from one's social position, focusing on psychological aspects in weight reduction might be a promising approach.
\end{abstract}

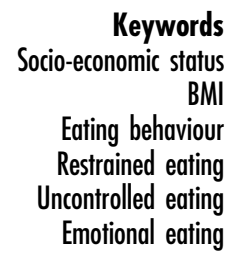

In Western countries, high standards of living provide good conditions for health, formally irrespective of one's social position. Despite these good theoretical conditions, there is an inverse gradient between socio-economic status (SES) and health. For example, in Germany, the estimated risk for diabetes mellitus type 2, heart disease or liver disease is twice as high for individuals with the lowest SES compared with individuals with the highest SES ${ }^{(1)}$.
Moreover, risk factors for common civilization diseases like physical inactivity and higher smoking prevalence, as well as less use of primary prevention services are more common in population groups with lower and lowest SES. This is also true for the risk factor high $\mathrm{BMI}^{(1,2)}$. For example, the German Health Update 2012 for the German population aged between 18 and 79 years revealed that $31.4 \%$ of women with the lowest SES were identified as 
overweight $\left(\mathrm{BMI} \geq 25.0 \mathrm{~kg} / \mathrm{m}^{2}\right)$ and another $23.6 \%$ as obese (BMI $\geq 30 \cdot 0 \mathrm{~kg} / \mathrm{m}^{2}$ ). But in women with the highest SES, the estimated overweight and obesity prevalence was much lower: $24.2 \%$ of the women were overweight and another $9.4 \%$ were obese ${ }^{(3)}$. For men, the findings are less clear. Nevertheless, obesity prevalence was also significantly higher in men with the lowest SES than in men with the highest SES $(20 \cdot 0 \mathrm{v} \cdot 14 \cdot 1 \%$ in the age group 18 to 79 years $)^{(3)}$. Overweight and obesity carry high social and economic burden. For example, in Germany, direct costs of obesity were estimated as $€ 29.39$ billion and the indirect costs as $€ 33.65$ billion per year ${ }^{(4)}$. Therefore, it is of utmost importance to identify the underlying links between SES and BMI, which might help to develop suitable prevention strategies against overweight and obesity.

One link between SES and BMI is eating behaviour, which differs between the SES groups. For example, higher consumption of sugar-sweetened beverages is more common in the lower and lowest SES groups ${ }^{(5,6)}$. Regarding the various interacting factors which lead to one's individual eating behaviour, including genetic, sociocultural and personal ones, there is no simple explanation for the differences in eating behaviour between SES groups. Therefore, we hypothesized that psychological factors of eating behaviour might be underlying factors between the inverse association of SES and BMI. Common and well-established psychological domains of eating behaviour are: (i) a domain which describes the degree of cognitive control in daily food intake (cognitive restrained, restrained eating); (ii) a domain which describes the loss of control over food intake (uncontrolled eating, disinhibition); and (iii) a domain which describes eating in response to emotional triggers like feeling anxious or depressed (emotional eating) ${ }^{(7-13)}$. The association between these psychological domains of eating behaviour and BMI are well studied. Especially the domains uncontrolled eating and emotional eating have been found to be associated with higher BMI values ${ }^{(14-17)}$. As eating behaviour has not only been found to be associated with BMI, but also with SES, in the present study we particularly aimed at investigating potential pathways between SES and the psychological eating behaviour domains 'restrained eating', 'uncontrolled eating' and 'emotional eating' and the BMI in the adult population. To the very best of our knowledge, this has not been done before.

\section{Methods}

\section{Etbics statement}

All participants provided written informed consent prior to their participation in the study. The study complies with the ethical standards of the Declaration of Helsinki and has been approved by the ethics committee of the University of Leipzig, Germany.

\section{Participants}

Data were derived from the large population-based LIFEAdult-Study that was conducted by the LIFE-Leipzig Research Center for Civilization Diseases in Leipzig, Germany (August 2011-November 2014). The LIFEAdult-Study aims to examine the prevalence, early onset markers, genetic predisposition and the role of lifestyle factors in major civilization diseases such as obesity, diabetes and CVD. The study design of the LIFE-Adult-Study has been described in detail elsewhere ${ }^{(18)}$. An age- and sex-stratified sample of residents of the city of Leipzig, Germany, was randomly selected from lists provided by the Leipzig registry office. The response rate was $33 \%$. Eating behaviour was completely assessed in 6657 participants. We excluded participants whose eating behaviour might have been influenced by symptoms of chronic inflammatory bowel disease, treatment of cancer disease, insulin treatment or intake of antipsychotic drugs ( $n$ 722). The present analysis was based on a final sample of 5935 participants aged 18-79 years.

\section{Data collection and assessment procedures}

All participants underwent a comprehensive assessment programme including a variety of physical and medical examinations, clinical interviews and standardized questionnaires. The assessments were conducted by trained study personnel at the LIFE research centre located on the premises of the University Hospital of Leipzig. For the present report, data were analysed from the following assessments.

\section{Eating behaviour}

Eating behaviour was assessed with the Fragebogen zum Essverhalten (FEV), the German version of the ThreeFactor Eating Questionnaire (TFEQ) ${ }^{(8,12)}$. The present analysis was based on a revised twenty-nine-item version of the TFEQ, which covers three domains of eating behaviour: 'uncontrolled eating', 'restrained eating' and 'emotional eating'. The internal consistency of the scales (Cronbach's $\alpha$ ) was stated as 0.802 for the domain of uncontrolled eating (contains fifteen items), $0 \cdot 840$ for the domain of restrained eating (contains eleven items) and 0.780 for the domain of emotional eating (contains three items) ${ }^{(17)}$.

As mentioned above, the domain of uncontrolled eating describes the tendency to lose control over food intake, the domain of restrained eating describes a permanent cognitive control of food intake order to lose or to control body weight, and the domain of emotional eating describes eating in response to emotional triggers like feeling anxious or depressed.

\section{Sociodemographic characteristics}

Sociodemographic information was obtained via a standardized interview, which included questions about the educational and occupational qualification of the participant, the net household income and actual occupational status. This information was used to assess the multidimensional 
SES-Index-Score in accordance with the approach used in the German Health Interview and Examination Survey for Adults ${ }^{(19)}$. The SES-Index-Score is based on the three domains of 'education', 'work' and 'income'. For each participant of the LIFE-Adult-Study, each of the three domains was rated between 1 point as the lowest possible value and 7 points as the highest possible value. The points of the three domains were then added to a final score (range 3-21 points) and participants were categorized into one of the three SES groups: 'low' (3-8 points), 'medium' (9-14 points) or 'high' (15-21 points).

\section{Anthropometry}

Body weight was measured with an electronic scale (SECA 701, Seca GmbH \& Co. KG) with a precision of $0.01 \mathrm{~kg}$. Body height was assessed by means of a stadiometer (SECA 240) to the nearest $0 \cdot 1 \mathrm{~cm}$. BMI was calculated as the weight in kilograms divided by the squared height in metres $\left(\mathrm{kg} / \mathrm{m}^{2}\right)$.

\section{Statistical analysis}

Statistical analyses were performed with the statistical software package IBM SPSS Statistics version 20.0, including the SPSS macro 'PROCESS' from Preacher and Hayes $^{(20)}$. All analysis employed an $\alpha$ level of 0.05 for statistical significance (two-tailed). Group differences were analysed using Mann-Whitney $U$ tests, $\chi^{2}$ tests and Kruskal-Wallis tests as appropriate.

To examine the pathways between SES, BMI and the eating behaviour domains, we conducted mediation analysis. For our analyses, we hypothesized that the psychological domains of eating behaviour (uncontrolled eating, restrained eating, emotional eating) mediate the association between SES-Index-Score and BMI. Previous analysis required differences in eating behaviour between men and women. Therefore, we conducted sex-stratified analysis ${ }^{(21)}$. We also used age as covariate, as age was found to be an influencing factor on eating behaviour ${ }^{(21)}$.

To detect the presence of mediation effects, we first examined the associations between the obtained variables. Therefore, we conducted Pearson's correlation analysis. Then we developed a parallel-mediation model to examine the potential direct, indirect and total effects of eating behaviour on the association between SES-IndexScore and BMI, controlled for covariates. Parallel mediation was used to simultaneously include the three eating behaviour domains in one model. A 'direct effect' describes the direct association between SES-Index-Score and BMI in the presence of the mediator(s). An 'indirect effect' describes the product of the paths between SESIndex-Score and BMI and between SES-Index-Score, the mediator(s) and BMI. The 'total effect' is the sum of the indirect and direct effect. Thus, a full mediation of the association between SES-Index-Score and BMI by eating behaviour would be present if there was an indirect effect via eating behaviour, but no direct effect of SES-Index-Score on BMI in the model. A partial mediation would be present if both the path between SES-Index-Score and BMI and the path between SES-Index-Score, eating behaviour and BMI was significant. To achieve scale similarity, we conducted a $z$-transformation of all included variables. A $z$-scale has an arithmetic mean of 0 and an SD of 1 , and allows the interpretation of regression coefficients more easily. Significance of the direct and indirect effects was tested with both the Sobel $z$-test and a bootstrapping procedure of about 10000 resamples. The bootstrap procedure providing $95 \% \mathrm{CI}$ is additionally recommended for significance testing as it respects irregularity of the sampling distribution and provides more power ${ }^{(20)}$.

\section{Results}

\section{Sample description}

Overall, analysis of the association between SES, eating behaviour and BMI was based on a sample of 5935 participants of the LIFE-Adult-Study. Characteristics of the study sample are shown in Table 1.

Table 2 presents a detailed overview on the association between the factors BMI and eating behaviour with SES. Group comparisons using Kruskal-Wallis tests revealed a higher mean BMI in lower SES groups, especially in women $\left(\chi^{2}\right.$ for men $=0.848, P=0.654 ; \chi^{2}$ for women $=$ $87 \cdot 137, P<0 \cdot 001)$. Additional information about the proportion of obesity in SES classes is given. The proportion of obesity was higher in lower and lowest SES in both men and women compared with the highest SES group. Values of restrained eating were higher in the higher SES groups in both men and women $\left(\chi^{2}\right.$ for men $=71 \cdot 304, P<0 \cdot 001$; $\chi^{2}$ for women $\left.=20 \cdot 241, P<0 \cdot 001\right)$. Values of uncontrolled eating and emotional eating did not differ between lowest and highest SES groups.

\section{Intercorrelation between SES-Index-Score, BMI, eating behaviour and the covariates}

As shown in Table 3, we found weak (Pearson's correlation coefficient $r<0.3)$ but significant associations between SES-Index-Score and BMI for both men and women. We also found weak associations between SES-Index-Score and all three eating behaviour domains as well as between BMI and the eating behaviour domains. Finally, we found a moderate $(r>0.4$ and $<0.7)$ association between the eating behaviour domains uncontrolled and emotional eating, and a weak association between the domains emotional and restrained eating.

\section{Parallel-mediation model}

We tested the pathways between SES-Index-Score, BMI and eating behaviour in separate parallel-mediation models for men and for women (see Figs. 1 and 2). In the models, the psychological eating behaviour domains uncontrolled eating, restrained eating and emotional 
Table 1 Main characteristics of and significance differences between the study sample of men and women ( $n$ 5935), LIFE-Adult-Study, Leipzig, Germany, August 2011-November 2014

\begin{tabular}{|c|c|c|c|c|c|}
\hline & \multicolumn{2}{|c|}{ Men (n 2905) } & \multicolumn{2}{|c|}{ Women (n 3030) } & \multirow[b]{2}{*}{$P$ value $\dagger$} \\
\hline & Mean or $\%$ & SD or $n$ & Mean or \% & SD or $n$ & \\
\hline \multicolumn{6}{|l|}{ Sociodemographic characteristics } \\
\hline \multicolumn{6}{|l|}{ Age } \\
\hline Age (years; range 18-79) & $54 \cdot 8$ & $12 \cdot 0$ & $53 \cdot 8$ & $11 \cdot 1$ & $<0.001$ \\
\hline \multicolumn{6}{|l|}{ Socio-economic status (SES) } \\
\hline SES-Index-Score (range 3-21) & $13 \cdot 5$ & 3.3 & $13 \cdot 1$ & $3 \cdot 1$ & $<0.001$ \\
\hline Low SES $(\%, n)$ & 4.8 & 139 & $4 \cdot 3$ & 129 & $<0.001$ \\
\hline Middle SES $(\%, n)$ & 49.5 & 1439 & $57 \cdot 4$ & 1740 & \\
\hline High SES $(\%, n)$ & $45 \cdot 7$ & 1327 & 38.3 & 1161 & \\
\hline \multicolumn{6}{|l|}{ Anthropometric measures } \\
\hline BMI $\left(\mathrm{kg} / \mathrm{m}^{2}\right)$ & $27 \cdot 4$ & 4.08 & $26 \cdot 8$ & 5.40 & $<0.001$ \\
\hline \multicolumn{6}{|l|}{ Eating behaviour domains $\ddagger$} \\
\hline Restrained eating score (range 0-15) & 4.6 & 3.52 & $6 \cdot 7$ & 3.90 & $<0.001$ \\
\hline Uncontrolled eating score (range $0-11$ ) & $2 \cdot 7$ & 2.53 & $3 \cdot 1$ & $2 \cdot 80$ & $<0.001$ \\
\hline Emotional eating score (range $0-3$ ) & 0.2 & 0.60 & 0.6 & 0.99 & $<0.001$ \\
\hline
\end{tabular}

†Group differences in continuous variables were analysed with the Mann-Whitney $U$ test, group differences in categorical variables were analysed with the $x^{2}$ test.

$\ddagger$ Higher scores indicate stronger characteristic values in the domains.

Table 2 Differences in BMI and eating behaviour scores according to socio-economic status (SES) for men and women ( $n$ 5935) aged 18-79 years, LIFE-Adult-Study, Leipzig, Germany, August 2011-November 2014

\begin{tabular}{|c|c|c|c|c|c|c|c|c|}
\hline & \multicolumn{2}{|c|}{ Low SES } & \multicolumn{2}{|c|}{ Medium SES } & \multicolumn{2}{|c|}{ High SES } & \multirow[b]{2}{*}{$x \dagger$} & \multirow[b]{2}{*}{$P$ value } \\
\hline & Mean & SD & Mean & SD & Mean & SD & & \\
\hline \multicolumn{9}{|l|}{ Men } \\
\hline BMI & 27.65 & $5 \cdot 17$ & 27.45 & $4 \cdot 14$ & $27 \cdot 27$ & 3.88 & 0.848 & 0.654 \\
\hline Obeseł & \multicolumn{2}{|c|}{28.80} & \multicolumn{2}{|c|}{24.30} & \multicolumn{2}{|c|}{$20 \cdot 90$} & $7 \cdot 289$ & 0.026 \\
\hline Restrained eating score§ & 3.36 & 3.26 & 4.22 & 3.42 & $5 \cdot 12$ & 3.56 & 71.304 & $<0.001$ \\
\hline Uncontrolled eating score§ & $2 \cdot 53$ & 2.48 & $2 \cdot 64$ & 2.50 & 2.87 & 2.56 & $7 \cdot 858$ & 0.020 \\
\hline Emotional eating score§ & 0.18 & 0.54 & 0.17 & 0.52 & 0.23 & 0.66 & 2.719 & 0.257 \\
\hline \multicolumn{9}{|l|}{ Women } \\
\hline BMI & 28.63 & $6 \cdot 13$ & $27 \cdot 37$ & 5.49 & $25 \cdot 79$ & 4.99 & $87 \cdot 137$ & $<0.001$ \\
\hline Obeseł & \multicolumn{2}{|c|}{36.40} & \multicolumn{2}{|c|}{$26 \cdot 70$} & \multicolumn{2}{|c|}{$17 \cdot 10$} & 47.917 & $<0.001$ \\
\hline Restrained eating score§ & $5 \cdot 26$ & 3.78 & $6 \cdot 76$ & 3.94 & 6.84 & 3.82 & $20 \cdot 241$ & $<0.001$ \\
\hline Uncontrolled eating score§ & 2.89 & 2.90 & 3.08 & 2.79 & $3 \cdot 21$ & 2.80 & 3.684 & 0.159 \\
\hline Emotional eating score $\S$ & 0.62 & 1.01 & 0.56 & 0.96 & 0.66 & 1.04 & 5.948 & 0.051 \\
\hline
\end{tabular}

†Group differences were analysed with the Kruskal-Wallis $H$ test.

fProportion of obesity in \%; obesity is classified as $\mathrm{BMI} \geq 30.0 \mathrm{~kg} / \mathrm{m}^{2(27)}$

$\S$ Higher scores indicate stronger characteristic values in the domains.

Table 3 Pearson's correlation coefficients between socio-economic status (SES; i.e. SES-Index-Score), BMI, eating behaviour and age for men and women ( $n$ 5935) aged $18-79$ years, LIFE-Adult-Study, Leipzig, Germany, August 2011-November 2014

\begin{tabular}{|c|c|c|c|c|c|}
\hline & BMI & RS & UC & EE & Age \\
\hline \multicolumn{6}{|l|}{ Men } \\
\hline SES & $-0.04^{*}$ & $0.15^{\star}$ & $0.07^{*}$ & $0.06^{*}$ & $0.01^{*}$ \\
\hline BMI & & $0.14^{*}$ & $0.32^{*}$ & $0.15^{\star}$ & $0.17^{*}$ \\
\hline $\mathrm{RS}$ & & & $0.00^{*}$ & $0.08^{*}$ & $0.22^{*}$ \\
\hline UC & & & & $0.37^{*}$ & $-0.14^{*}$ \\
\hline $\mathrm{EE}$ & & & & & $-0.07^{*}$ \\
\hline Age & & & & & \\
\hline \multicolumn{6}{|l|}{ Women } \\
\hline SES & $-0.18^{*}$ & $0.06^{*}$ & $0.01^{*}$ & $0.04^{*}$ & $-0.11^{*}$ \\
\hline BMI & & $0.15^{\star}$ & $0.25^{\star}$ & $0.22^{*}$ & $0.23^{*}$ \\
\hline $\mathrm{RS}$ & & & $-0.01^{*}$ & $0.09^{*}$ & $0.20^{\star}$ \\
\hline UC & & & & $0.48^{*}$ & $-0.14^{*}$ \\
\hline $\begin{array}{l}\text { EE } \\
\text { Age }\end{array}$ & & & & & $-0.07^{*}$ \\
\hline Age & & & & & \\
\hline
\end{tabular}

RS, restrained eating; UC, uncontrolled eating; EE, emotional eating. ${ }^{*} P<0.05$. eating were included as potential mediators between SESIndex-Score and BMI. Age was included as a covariate. Results of the mediation models are presented in Table 4.

In both men and women, we found a significant direct path between SES-Index-Score and BMI. The effect was estimated as $\beta=-0.07$ (SE $0.01 ; P<0.001$ ) for men and $\beta=-0.19$ (se $0.02 ; P<0.001$ ) for women. Regarding the paths between SES-Index-Score, eating behaviour variables and BMI in detail, we found differences between the models for men and women. For men, we found direct significant paths between SES-Index-Score and all three eating behaviour domains (restrained eating: $\beta=0.13$ (sE 0.02), $P<0.001$; uncontrolled eating: $\beta=0.06$ (sE 0.02 ), $P<0.05$; emotional eating: $\beta=0.04$ (se 0.01 ),$P<0.05$ ). The paths between the eating behaviour domains and BMI were also significant (restrained eating: $\beta=0.09$ (SE 0.02), $P<0.001$; uncontrolled eating: $\beta=0.30$ (se 0.02 ),$P<0.001$; 


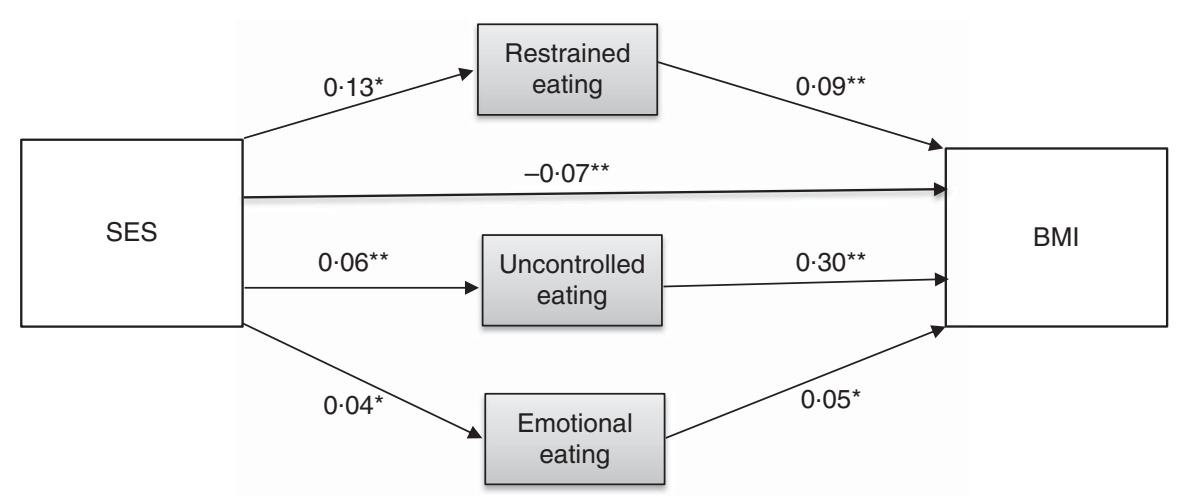

Fig. 1 Parallel-mediation model between socio-economic status (SES; i.e. SES-Index-Score), BMI and psychological domains of eating behaviour for men ( $n$ 2905) aged 18-79 years, LIFE-Adult-Study, Leipzig, Germany, August 2011-November 2014. ${ }^{*}$ Significant path ( $z$-test) with $a$ level of $<0.05$; ${ }^{* *}$ significant path $(z$-test) with $a$ level of $<0.001$

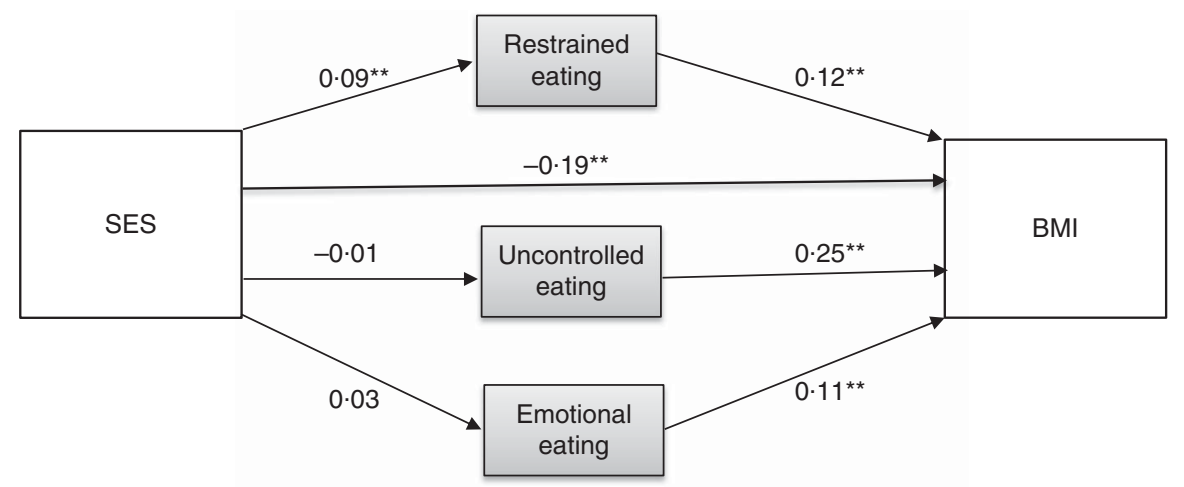

Fig. 2 Parallel-mediation model between socio-economic status (SES; i.e. SES-Index-Score), BMI and psychological domains of eating behaviour for women ( $n$ 3030) aged 18-79 years, LIFE-Adult-Study, Leipzig, Germany, August 2011-November 2014. ${ }^{* \star}$ Significant path ( $z$-test) with $a$ level of $<0.001$

Table 4 Results from the mediation models with direct, indirect and total effects of socio-economic status (SES; i.e. SES-Index-Score) and eating behaviour on BMI for men $\left(r^{2}=0.032\right)$ and women $\left(r^{2}=0.075\right)$ aged $18-79$ years, LIFE-Adult-Study, Leipzig, Germany, August 2011-November 2014

\begin{tabular}{|c|c|c|c|c|c|}
\hline \multirow[b]{2}{*}{ Men } & \multirow[t]{2}{*}{$\beta$} & \multirow[t]{2}{*}{ SE } & \multirow[t]{2}{*}{$P$ value (normal theory) } & \multicolumn{2}{|c|}{ Bootstrap $95 \% \mathrm{Cl}$} \\
\hline & & & & & \\
\hline Total effect $†$ & -0.033 & 0.015 & 0.032 & -0.062 & -0.003 \\
\hline $\begin{array}{l}\text { Direct effect‡ } \\
\text { Indirect effect§ }\end{array}$ & -0.066 & 0.014 & $<0.001$ & -0.094 & -0.038 \\
\hline Restrained eating score & 0.012 & 0.003 & $<0.001$ & 0.008 & 0.018 \\
\hline Uncontrolled eating score & 0.016 & 0.005 & 0.003 & 0.009 & 0.030 \\
\hline Emotional eating score & 0.002 & 0.001 & 0.066 & 0.000 & 0.006 \\
\hline \multicolumn{6}{|l|}{ Women } \\
\hline Total effect† & -0.178 & 0.020 & $<0.001$ & -0.217 & -0.138 \\
\hline $\begin{array}{l}\text { Direct effect‡ } \\
\text { Indirect effect§ }\end{array}$ & -0.191 & 0.019 & $<0.001$ & -0.228 & -0.153 \\
\hline Restrained eating score & 0.011 & 0.003 & 0.002 & 0.006 & 0.027 \\
\hline Uncontrolled eating score & -0.004 & 0.005 & 0.909 & -0.010 & 0.009 \\
\hline Emotional eating score & 0.003 & 0.003 & 0.144 & -0.001 & 0.001 \\
\hline
\end{tabular}

†Total effect $=$ direct effect + indirect effects.

†Direct effect $=$ path between SES-Index-Score and BMI in presence of mediators.

SIndirect effect = paths between SES-Index-Score, eating behaviour domains and BMI.

emotional eating: $\beta=0.05$ (se 0.02 ), $P<0.05$ ). For women, there were no significant paths between SES-Index-Score and the eating behaviour domains uncontrolled eating and emotional eating, but between SES-Index-Score and the domain restrained eating $(\beta=0.09$ (SE 0.02), $P<0 \cdot 001$ ).
Similarly to the results for men, however, all three eating behaviour variables showed significant paths to BMI also in women (restrained eating: $\beta=0 \cdot 12$ (SE 0.02), $P<0 \cdot 001$; uncontrolled eating: $\beta=0.25$ (sE 0.02 ), $P<0.001$; emotional eating: $\beta=0 \cdot 11$ (sE $0 \cdot 02$ ), $P<0 \cdot 001$ ). The total effect 
between SES-Index-Score and BMI regarding eating behaviour variables was estimated as $\beta=-0.03$ (SE 0.02 ; $95 \%$ CI $-0.062,-0.003$ ) for men and as $\beta=-0.18$ (SE 0.02 ; $95 \% \mathrm{CI}-0 \cdot 217,-0 \cdot 138$ ) for women.

Overall, the mediation models indicated a partial mediation by psychological eating behaviour domains in both men and women. In men, restrained eating $(\beta=0.012$ (SE 0.003); 95\% CI 0.008, 0.018) and uncontrolled eating ( $\beta=0.016$ (sE 0.005); 95\% CI 0.009, 0.030) mediated the association between SES-Index-Score and BMI. In women, a mediation effect of restrained eating between SES-IndexScore and BMI was found ( $\beta=0.011$ (SE 0.003); $95 \% \mathrm{CI}$ $0 \cdot 006,0 \cdot 027)$. Emotional eating did not act as a mediator between SES-Index-Score and BMI in both men and women; results rather showed an independent impact of emotional eating on BMI.

\section{Discussion}

In the present study, we aimed to investigate potential pathways from SES to BMI in the adult population, considering the psychological eating behaviour domains restrained eating, uncontrolled eating and emotional eating as potential mediators stratified for sex. We hypothesized that psychological domains of eating behaviour mediate the association between SES and BMI.

In detail, the eating behaviour factor restrained eating was found to attenuate the indirect association between SES-Index-Score and BMI. This means that low restrained eating might explain higher BMI in lower SES. Higher SES is associated with higher restrained eating. Importantly, restrained eating might be a strong result of individual health considerations. It has been shown that highly educated individuals have higher health considerations, which might in turn lead to restrained eating to stop weight gain, to maintain weight or to lose weight ${ }^{(22)}$. Thus, this result corroborates health awareness as one important component in weight reduction programmes or in part of health education in general.

We also found a significant mediation effect of uncontrolled eating on the association between SES-Index-Score and BMI. However, as this effect was found only in men, and moreover was only small, further studies may be waited for to confirm this finding. Nevertheless, as the direct association between uncontrolled eating and BMI is well studied, coping with a loss of control in food intake might be part of a weight reduction programme ${ }^{(14-17)}$.

Emotional eating did not mediate the association between SES-Index-Score and BMI in both men and women. Thus, our analysis suggests that emotional eating is rather directly associated with BMI - independent of SES. The positive association between emotional eating and BMI was also found in several other studies and underlines the importance of this eating behaviour domain for understanding weight gain ${ }^{(13,15-17,23,24)}$. Therefore, alternative coping strategies for emotions might be a very useful approach in attempts to lose or to maintain weight independent from one's social position.

However, regarding the low total effect of all the eating behaviour domains on the association between SES-Index-Score and BMI in mediation analyses, our study, in general, does not indicate a strong mediation effect of psychological domains of eating behaviour. As we were able to focus only on three domains of eating behaviour, more research is encouraged to investigate further potentially important aspects (e.g. social norms, habits, food marketing strategies) in mediation analyses of the association between SES and BMI. Nevertheless, independent from one's social position, focusing on psychological aspects in a weight reduction process might be a promising approach. Therefore, the identification of internal or external food stimuli, such as food advertising, might help to develop individual coping strategies in a weight reduction process. For example, a review from Mantzios et al. found that 'mindfulness eating' might reduce emotional as well as uncontrolled eating, which are both associated with higher BMI values ${ }^{(17,25)}$.

Our study is not without limitations. First, there is the probability of selection bias because of a relatively low response rate and some missing information about eating behaviour. Second, individuals with lower SES were under-represented, which might have caused bias, too. Finally, self-reported data of eating behaviour might have also led to some bias, such as over- or under-reporting. Nevertheless, irrespective of these limitations, we found that the association between SES and BMI was partly mediated by eating behaviour.

However, the association between SES and BMI, in general, seems to be complex and there might be more than one pathway involved. To the very best of our knowledge, our study is the first that examined a pathway via the psychological domains of eating behaviour. So far, few studies also examined other potential psychological aspects in mediation analysis. Beydoun et al., for example, found that depressive symptoms may mediate the inverse association between SES and obesity in women ${ }^{(26)}$.

\section{Acknowledgements}

Acknowledgements: The authors would like to thank all participants and the staff at LIFE-Leipzig Research Center for Civilization Diseases. Financial support: This publication is supported by LIFE-Leipzig Research Center for Civilization Diseases, Universität Leipzig. LIFE is funded by the European Union through the European Regional Development Fund (ERDF) and by the Free State of Saxony within the framework of the excellence initiative. F.S.T. has been supported by LIFE-Leipzig Research Center for Civilization Diseases, Universität Leipzig. Her collaboration within LIFE was funded by means of the 
European Social Fund and the Free State of Saxony. Y.B. is funded by IFB Adiposity Diseases. IFB Adiposity Diseases is supported by the Federal Ministry of Education and Research (BMBF), Germany (grant number FKZ: 01EO1501). Conflict of interest: None of the authors has any conflict of interest to declare. Authorship: M.L., J.T., C.E., A.V., M.S. and S.G.R.-H. conceived and designed the study. A.L. performed the statistical analysis, interpreted the data and drafted the manuscript. T.L. and F.S.T. helped to interpret the data and to perform the statistical analysis and were involved in drafting and revising the manuscript critically for important contents. A.P. advised in statistical analysis. S.G.R.-H. supervised the study. She as well as M.L., J.T., C.E., A.V., M.S., C.L.-S., A.P., P.K., Y.B., J.B., A.T. and A.H. revised the manuscript critically for important contents. All authors approved the final version. Ethics of buman subject participation: This study was conducted according to the guidelines laid down in the Declaration of Helsinki and all procedures involving human subjects were approved by the ethics committee of the University of Leipzig, Germany. All subjects provided written informed consent prior to their participation in the study.

\section{References}

1. Lampert T, Kroll LE, Lippe E von der et al. (2013) Socioeconomic status and health: results of the German Health Interview and Examination Survey for Adults (DEGS1). Bundesgesundheitsblatt Gesundheitsforschung Gesundheitsschutz 56, 814-821.

2. Weyers S, Dragano N, Richter M et al. (2010) How does socio economic position link to health behaviour? Sociological pathways and perspectives for health promotion. Glob Health Promot 17, 25-33.

3. Robert Koch-Institut (editor) (2012) Daten und Fakten: Ergebnisse der Studie 'Gesundheit in Deutschland aktuell 2010': Gesundheitsberichterstattung-Hefte. Berlin: Robert Koch-Institut.

4. Effertz T, Engel S, Verheyen F et al. (2015) The costs and consequences of obesity in Germany: a new approach from a prevalence and life-cycle perspective. Eur J Health Econ 17, 1141-1158.

5. Malik VS, Schulze MB \& Hu FB (2006) Intake of sugarsweetened beverages and weight gain: a systematic review. Am J Clin Nutr 84, 274-288.

6. Weyers S, Fekete C, Dragano N et al. (2013) Education, selected nutrition attitudes and nutrition behaviour. Prävention und Gesundheitsförderung 8, 289-294.

7. Ganley RM (1988) Emotional eating and how it relates to dietary restraint, disinhibition, and perceived hunger. Int $J$ Eat Disord 7, 635-647.

8. Stunkard AJ \& Messick S (1985) The three-factor eating questionnaire to measure dietary restraint, disinhibition and hunger. J Psychosom Res 29, 71-83.

9. Hyland ME, Irvine SH, Thacker C et al. (1989) Psychometric analysis of the Stunkard-Messick Eating Questionnaire (SMEQ) and Comparison with the Dutch Eating Behavior Questionnaire (DEBQ). Curr Psychol 8, 228-233.

10. Karlsson J, Persson LO, Sjostrom L et al. (2000) Psychometric properties and factor structure of the Three-Factor
Eating Questionnaire (TFEQ) in obese men and women. Results from the Swedish Obese Subjects (SOS) study. Int J Obes Relat Metab Disord 24, 1715-1725.

11. Mazzeo SE, Aggen SH, Anderson C et al. (2003) Investigating the structure of the eating inventory (three-factor eating questionnaire): a confirmatory approach. Int J Eat Disord 34, 255-264.

12. Pudel V \& Westenhoefer J (1989) Fragebogen zum Eßverhalten. Göttingen: Hogrefe.

13. van Strien T, Frijters JER, Bergers GPA et al. (1986) The Dutch Eating Behavior Questionnaire (DEBQ) for assessment of restrained, emotional, and external eating behavior. Int J Eat Disord 5, 295-315.

14. French SA, Epstein LH, Jeffery RW et al. (2012) Eating behavior dimensions. Associations with energy intake and body weight. A review. Appetite 59, 541-549.

15. Geliebter A \& Aversa A (2003) Emotional eating in overweight, normal weight, and underweight individuals. Eat Behav 3, 341-347.

16. Koenders PG \& van Strien T (2011) Emotional eating, rather than lifestyle behavior, drives weight gain in a prospective study in 1562 employees. J Occup Environ Med 53, 1287-1293.

17. Loffler A, Luck T, Then FS et al. (2015) Eating behaviour in the general population: an analysis of the factor structure of the German version of the Three-Factor-EatingQuestionnaire (TFEQ) and its association with the body mass index. PLoS One 10, e0133977.

18. Loeffler M, Engel C, Ahnert P et al. (2015) The LIFE-AdultStudy: objectives and design of a population-based cohort study with 10,000 deeply phenotyped adults in Germany. BMC Public Health 15, 691.

19. Lampert T, Kroll L, Müters S et al. (2013) Messung des sozioökonomischen Status in der Studie zur Gesundheit Erwachsener in Deutschland (DEGS1). Bundesgesundheitsblatt Gesundheitsforschung Gesundheitsschutz 56, 631-636.

20. Preacher KJ \& Hayes AF (2008) Asymptotic and resampling strategies for assessing and comparing indirect effects in multiple mediator models. Behav Res Methods $\mathbf{4 0}$, 879-891.

21. Löffler A, Luck T, Then FS et al. (2015) Age- and genderspecific norms for the German version of the Three-Factor Eating-Questionnaire (TFEQ). Appetite 91, 241-247.

22. Ball K, Crawford D \& Mishra G (2006) Socio-economic inequalities in women's fruit and vegetable intakes: a multilevel study of individual, social and environmental mediators. Public Health Nutr 9, 623-630.

23. Konttinen H, Mannisto S, Sarlio-Lahteenkorva S et al. (2010) Emotional eating, depressive symptoms and self-reported food consumption. A population-based study. Appetite $\mathbf{5 4}$, 473-479.

24. Horstmann A, Kovacs P, Kabisch S et al. (2013) Common genetic variation near MC4R has a sex-specific impact on human brain structure and eating behavior. PLoS One $\mathbf{8}$, e74362.

25. Mantzios M \& Wilson JC (2015) Mindfulness, eating behaviours, and obesity: a review and reflection on current findings. Curr Obes Rep 4, 141-146.

26. Beydoun MA, Kuczmarski MTF, Mason MA et al. (2009) Role of depressive symptoms in explaining socioeconomic status disparities in dietary quality and central adiposity among US adults: a structural equation modeling approach. Am J Clin Nutr 90, 1084-1095.

27. World Health Organization (2000) Obesity: Preventing and Managing the Global Epidemic. Report of a WHO Consultation. WHO Technical Report Series no. 894. Geneva: WHO. 